\title{
Global attracting set for a class of delayed Hopfield neural networks

\author{
Qinghua Zhou ${ }^{1, \text { a }}$, Li Wan ${ }^{2, b}$
}

${ }^{1}$ School of Mathematics and Statistics, Zhaoqing University, Zhaoqing 526061, China

${ }^{2}$ School of Mathematics and Computer Sciences, Wuhan Textile University, Wuhan 430073, China

azqhmath@126.com, bwanlinju@aliyun.com

Keywords: Hopfield neural networks; Global attracting set; Integral inequality.

Abstract. The asymptotic behaviors of a class of delayed Hopfield neural networks are investigated. By applying the property of nonnegative matrix and an integral inequality, some novel sufficient conditions are derived to ensure the existence of the global attracting set and the stability in a Lagrange sense for the considered networks. Finally, a numerical example is given to demonstrate the effectiveness of our theoretical result. Our criteria are easily tested by Matlab LMI Toolbox.

\section{Introduction Preliminaries}

The well-known Hopfield neural networks were firstly introduced by Hopfield in early 1980s. Since then, both the mathematical analysis and practical applications of Hopfield neural networks have gained considerable research attention. Hopfield neural networks have already been successfully applied in many different areas such as combinatorial optimization, knowledge acquisition and pattern recognition. Such applications strongly depend on the stability of the equilibrium point of the networks [1-6]. But the equilibrium point sometimes does not exist in many real physical systems. Therefore, a number of scholars pay their attention to study the attracting sets of the neural networks

with delays and the estimate for the domain of attraction of the origin is given [7-10].

On the other hand, an inequality technique is an important researching tool in studying differential equation, see [11-13]. However, the equalities mentioned above are ineffective for the existence of the global attracting set of the following Hopfield neural networks with delays

$$
\left\{\begin{array}{l}
\dot{x}_{i}(t)=-a_{i}(t) x_{i}(t)+\sum_{j=1}^{n} b_{i j}(t) f_{j}\left(x_{j}(t)\right)+\sum_{j=1}^{n} c_{i j}(t) f_{j}\left(\dot{x}_{j}\left(t-\tau_{j}\right)\right)+I_{i}(t) \\
x_{i}(s)=\varphi_{i}(s), s \in\left(-\infty, t_{0}\right], i=1,2, \cdots, n
\end{array}\right.
$$

where $x_{i}(t)$ is the activations of the ith neuron in $F_{x}, a_{i}(t) \geq 0$ denotes the passive decay rate, $b_{i j}(t)$ and $c_{i j}(t)$ represent the weight coefficient of the neurons respectively. $f_{j}$ is activation function, $I_{i}(t)$ is the external input. $\tau_{j}$ is the transmission delay with $\tau=\max _{1 \leq j \leq n}\left\{\tau_{j}\right\} \leq 1 . \varphi_{i}(\bullet)$ denotes real-valued continuous function defined on $\left(-\infty, t_{0}\right]$.

So we will give an integral inequality which is effective for system (1.1) and derive the sufficient conditions to ensure the existence of the global attracting set and the stability in a Lagrange sense for system (1.1).

\section{Preliminaries}

Throughout this paper, $E_{n}$ denotes $n \times n$-dimensional unit matrix. $\mathbb{R}$ is the set of real numbers and $\mathbb{R}_{+}=[0,+\infty)$, and the symbol $\mathbb{R}^{n}$ stands for the $n$-dimensional Euclidean space. For square matrix $A, A^{-1}$ denotes its inverse, and $\rho(A)$ is its spectral radius. $A \geq 0$ means that $A$ is called a 
nonegative matrix. $C(X, Y)$ denotes the space of continuous mappings from the topological space $X$ to the topological space $Y$. We denote $a(t)=\operatorname{diag}\left\{a_{1}(t), \cdots, a_{n}(t)\right\}, \bar{I}(t)=\left(\left|I_{1}(t)\right|, \cdots,\left|I_{n}(t)\right|\right)^{T}$, $b(t)=\left(b_{i j}(t)\right)_{n \times n}, \quad c(t)=\left(c_{i j}(t)\right)_{n \times n}, \quad B(t)=\left(\left|b_{i j}(t)\right|\right)_{n \times n}, \quad C(t)=\left(\left|c_{i j}(t)\right|\right)_{n \times n}$.

Before finishing this section, we introduce the following assumptions, definitions and lemmas.

$\left(A_{1}\right)$ For any $x_{j} \in \mathbb{R}, j \in \mathbb{N}$, there exists a constant $l_{j} \geq 0$, such that $\left|f_{j}\left(x_{j}\right)\right| \leq l_{j}\left|x_{j}\right|$, $L=\operatorname{diag}\left\{l_{1}, \cdots, l_{n}\right\}$.

$\left(A_{2}\right)$ For $t \geq t_{0}$, there exists a constant matrix $\Sigma \geq 0$, such that $e^{-\int_{s}^{t} a(v) d v} C(s) L \leq \Sigma$.

$\left(A_{3}\right)$ For $t \geq t_{0}$, there exist a nonnegative constant matrix $\Pi$ and a constant vector $I \geq 0$, such that $\int_{t_{0}}^{t} e^{-\int_{s}^{t} a(v) d v} B(s) L d s \leq \Pi, \int_{t_{0}}^{t} e^{-\int_{s}^{t} a(v) d v} \bar{I}(s) d s \leq I$.

$\left(A_{4}\right) \bar{\Pi} \triangleq \Sigma /(1-\tau)+\Pi \geq 0, \quad \rho(\bar{\Pi}) \leq 1$.

$\left(A_{5}\right) k_{i} \triangleq \inf _{t_{0} \leq s \leq t} \int_{s}^{s+\theta} a_{i}(v) d v>0$, for some $\theta>0, i=1,2, \cdots, n$.

Definition 1. System (1.1) is uniformly bounded with respect to partial state $x(t)$, if for any constant $\varepsilon>0, t_{0} \geq 0$, there exists constant $\delta(\varepsilon)>0$, such that $\left\|x\left(t, t_{0}, \varphi\right)\right\| \leq \varepsilon$ for all $t \geq t_{0}$ and $\sup _{t_{0}-\tau \leq s \leq t_{0}}\|\varphi(s)\|<\delta(\varepsilon)$.

Definition 2. $\Omega$ is said to be a global attracting set of system (1.1), if there exists a compact set $\Omega \subset \mathbb{R}^{n}$, such that for $\forall \varphi \in C\left(\left(-\infty, t_{0}\right], \mathbb{R}^{n}\right)$, $\limsup _{t \rightarrow+\infty} d(x(t), \Omega)=0$, where $x(t)=x\left(t, t_{0}, \varphi\right)$, $d(x(t), \Omega)$ denotes the distance of $x(t)$ to $\Omega$ in $\mathbb{R}^{n}$.

Definition 3. ([12]) $f(t, s) \in U C_{t}$ means that $f \in C\left(\mathbb{R}_{+}, \mathbb{R}_{+}\right)$and for any given $\eta$ and any $\varepsilon>0$, there exist constants $\beta, T$ and $\alpha$ such that for any $t \geq \alpha, \int_{\eta}^{t} f(s) d s \leq \beta, \int_{\eta}^{t-T} f(s) d s \leq \varepsilon$.

Lemma 1. ( [14]) For any nonegative matrix $A \geq 0$, if $\rho(A)<1$, then $(E-A)^{-1} \geq 0$.

Lemma 2. Let $G\left(t, t_{0}\right) \in C\left(\mathbb{R} \times \mathbb{R}, \mathbb{R}_{+}^{n}\right), B \in \mathbb{R}_{+}^{n \times n}, Q(t, s) \in C\left(\mathbb{R} \times \mathbb{R}, \mathbb{R}_{+}^{n \times n}\right), I=\left(i_{1}, \cdots, i_{n}\right)^{T} \geq 0$, $\varphi(t) \in C\left(\left(-\infty, t_{0}\right], \mathbb{R}_{+}^{n}\right), \quad \alpha_{1}$ is a constant, $\|x(t)\|_{\tau}=\left(\left\|x_{1}(t)\right\|_{\tau}, \cdots,\left\|x_{n}(t)\right\|_{\tau}\right),\left\|x_{i}(t)\right\|_{\tau}=\max _{0 \leq s \leq \tau}$ $\left|x_{i}(t-s)\right|$ and $x(t) \in C\left(\mathbb{R}, \mathbb{R}_{+}^{n}\right)$ is a solution of the delay integral inequality

$$
\left\{\begin{array}{l}
x(t) \leq G\left(t, t_{0}\right)+B\|x(t)\|_{\tau}+\int_{\alpha_{1}}^{t} Q(t, s)\|x(s)\|_{\tau} d s+I \\
x(t)=\varphi(t), t \neq t_{0} .
\end{array}\right.
$$

Then there exists a constant vector $M>0$ such that for $t \geq t_{0}, x(t)<\left(E_{n}-\bar{P}\right)^{-1}(M+I)$, provided that the following conditions are satisfied:

(i) $G \triangleq \sup _{t_{0} \leq s \leq+\infty} G\left(s, t_{0}\right)$, and there exists an nonnegative constant matric $P$ such that for $t \geq t_{0}, \int_{\alpha_{1}}^{t} Q(t, s) d s \leq P$.

(ii) $\stackrel{-}{P}=P+B$ and $\rho(\bar{P})<1$.

Proof. From the condition (ii) and Lemma $1,\left(E_{n}-\bar{P}\right)^{-1}$ exists and $\left(E_{n}-\bar{P}\right)^{-1}$. Then there exists a constant vector

$\bar{G} \geq G$, such that $\varphi(t)<\left(E_{n}-\bar{P}\right)^{-1} M, \forall t \in\left(-\infty, t_{0}\right]$. 
We assume $x(t)<\left(E_{n}-\bar{P}\right)^{-1}(M+I)$ is not true. Without loss of generality, there must be a constant $t_{1}>t_{0}$ and an integer $\alpha \in\{1, \cdots, n\}$ such that $x_{\alpha}\left(t_{1}\right)=\left\{\left(E_{n}-\bar{P}\right)^{-1}(M+I)\right\}_{\alpha}, t \leq t_{1}$, $x(t) \leq\left(E_{n}-\bar{P}\right)^{-1}(M+I)$, where $\{\bullet\}_{i}$ denotes the $i$ th component of vector $\{\bullet\}$.

From the condition (i), $G\left(t, t_{0}\right)<M$ and (2.1), we obtain

$$
\begin{aligned}
x_{\alpha}\left(t_{1}\right) & \leq\left\{G\left(t_{1}, t_{0}\right)+B\left\|x\left(t_{1}\right)\right\|_{\tau}+\int_{\alpha_{1}}^{t_{1}} Q(t, s)\|x(s)\|_{\tau} d s+I\right\}_{\alpha} \\
& \leq\left\{M+\left[B+\int_{\alpha_{1}}^{t_{1}} Q(t, s) d s\right]\left(E_{n}-\bar{P}\right)^{-1}(M+I)+I\right\}_{\alpha} \\
& \leq\left\{M+\bar{P}\left(E_{n}-\bar{P}\right)^{-1}(M+I)+I\right\}_{\alpha}=\left\{\left(E_{n}-\bar{P}\right)^{-1}(M+I)\right\}_{\alpha},
\end{aligned}
$$

which contradicts the equality $x_{\alpha}\left(t_{1}\right)=\left\{\left(E_{n}-\bar{P}\right)^{-1}(M+I)\right\}_{\alpha}$. The proof is completed.

\section{Main Results}

Theorem 1. System (1.1) is uniformly bounded provided that the assumptions $\left(A_{1}\right)-\left(A_{4}\right)$ hold.

Proof. Using the variation of parameter formula and $\left(A_{1}\right)$, we obtain for any $t>t_{0}$, $|x(t)| \leq e^{-\int_{s}^{t} a(v) d v}\left|\varphi\left(t_{0}\right)\right|+\int_{t_{0}}^{t} e^{-\int_{s}^{t} a(v) d v} B(s) L\|x(s)\|_{\tau} d s+\int_{t_{0}}^{t} e^{-\int_{s}^{t} a(v) d v} C(s) L\|\dot{x}(s-\tau)\| d s+\int_{t_{0}}^{t} e^{-\int_{s}^{t} a(v) d v} I(s) d s$.

Form the assumption $\left(A_{2}\right)$, we have

$$
\int_{t_{0}}^{t} e^{-\int_{s}^{t} a(v) d v} C(s) L\|\dot{x}(s-\tau)\| d s \leq \sum\left(\|x(t)\|_{\tau}+\left|\varphi\left(t_{0}-\tau\right)\right|\right) /(1-\tau) .
$$

From (3.1) and (3.2), we have

$|x(t)| \leq e^{-\int_{s}^{t} a(v) d v}\left|\varphi\left(t_{0}\right)\right|+\frac{\sum}{1-\tau}\left|\varphi\left(t_{0}-\tau\right)\right|+\frac{\sum}{1-\tau}\|x(s)\|_{\tau} d s+\int_{t_{0}}^{t} e^{-\int_{s}^{t} a(v) d v} B(s) L\|x(s)\|_{\tau} d s+\int_{t_{0}}^{t} e^{-\int_{s}^{t} a(v) d v} \bar{I}(s) d s$.

By (3.3), $\left(A_{3}\right),\left(A_{4}\right)$ and Lemma 2, there exists a constant vector , such that

$$
|x(t)|<\left(E_{n}-\bar{\Pi}\right)^{-1}(\bar{\Pi}+K+I), \forall t>t_{0} .
$$

which implies that the solution of (1.1) is uniformly bounded with respect to partial states $x(t)$.

Theorem 2. Suppose that the assumptions $\left(A_{1}\right)-\left(A_{5}\right)$ hold. Then the set

$$
\Omega=\left\{\mu \in \mathbb{R}^{n}|| \mu \mid \leq\left(E_{n}-\bar{\Pi}\right)^{-1}\left(\Pi+\frac{\Sigma}{1-\tau}\left|\varphi\left(t_{0}-\tau\right)\right|+I\right)\right\}
$$

is a global attracting set of system (1.1), which implies that system (1.1) is globally stable in a Lagrange sense.

Proof.

From Theorem 1, there exists a nonnegative constant vector $\delta \in \mathbb{R}^{n}$ such that

$$
\lim _{t \rightarrow+\infty} \sup |x(t)|=\delta \leq\left(E_{n}-\bar{\prod}\right)^{-1}\left(\bar{\prod}+K+I\right) .
$$

Next, we will show that $\delta \in \Omega$.

From $\left(A_{5}\right)$ and Definition $3, \quad$ it is easy to see $e^{-\int_{s}^{t} a_{i}(v) d v} \in U C_{t}, e^{-\int_{s}^{t} a_{i}(v) d v} a_{i}(s) \in U C_{t} \quad$ for $i=1,2, \cdots, n$. Then, for any $\gamma>0$ and $\varepsilon=(1,1, \cdots, 1) \in \mathbb{R}^{n}$, there exists a positive number $A$ and constant matrix $R$ such that for any $t>t_{0}+A$, 


$$
e^{-\int_{t_{0}}^{t} a(v) d v}\left|\varphi\left(t_{0}\right)\right|<\frac{\gamma \varepsilon}{4}, \int_{t-A}^{t} e^{-\int_{s}^{t} a(v) d v} d s \leq R, \int_{t_{0}}^{t-A} e^{-\int_{s}^{t} a(v) d v} B(s) L\left(E_{n}-\bar{\Pi}\right)^{-1}(\bar{\Pi}+K+I) d s<\frac{\gamma \varepsilon}{4} .
$$

So there exists sufficient large $t_{2} \geq t_{0}+2 A$, such that

$$
\|x(t)\|_{\tau}<\delta+\gamma \varepsilon, t \geq t_{2} .
$$

Thus, from $\left(A_{3}\right)$, (3.3) and (3.6)-(3.7), it is easy to obtain for $t \geq t_{2}$,

$$
|x(t)| \leq \gamma \varepsilon+\frac{\sum}{1-\tau}\left|\varphi\left(t_{0}-\tau\right)\right|+\bar{\Pi}(\delta+\gamma \varepsilon)+\Pi+I .
$$

Together with (3.5), there is $t_{3} \geq t_{2}$ such that $\left|x\left(t_{3}\right)\right|>\delta-\gamma \varepsilon$. Letting $\gamma \rightarrow 0$, we obtain

$$
\delta<\left(E_{n}-\bar{\Pi}\right)^{-1}\left(\Pi+\frac{\Sigma}{1-\tau}\left|\varphi\left(t_{0}-\tau\right)\right|+I\right),
$$

that is, $\delta \in \Omega$. Therefore, the set $\Omega$ is a global attracting set of system (1.1), which also shows that system (1.1) is globally stable in a Lagrange sense.

Example 1. Consider system (1.1) with

$$
a(t)=\left(\begin{array}{ll}
1 & 0 \\
0 & 2
\end{array}\right), \quad b(t)=\left(\begin{array}{cc}
0.7121 \sin t & 0 \\
0.0667 & 0.1167
\end{array}\right), \quad c(t)=\left(\begin{array}{cc}
0.0167 & 0 \\
0 & 0.0333
\end{array}\right)
$$

$\forall t \geq 0$, and $\tau>0$ is a constant, $f(y(t))=y(t), I(t)=(\sin t, \arctan t)^{T}$. Obviously, $L=E_{n}$ and by computing, we know $\int_{0}^{t} e^{-(t-s)}|\sin s| d s \leq 0.8660, \sum=E_{n}$. Then one has

$$
\sum=\left(\begin{array}{cc}
0.0167 & 0 \\
0 & 0.0333
\end{array}\right), \quad \Pi=\left(\begin{array}{cc}
0.9666 & 0 \\
0.0333 & 0.2167
\end{array}\right), \quad I=\left(\begin{array}{l}
0.8660 \\
0.7854
\end{array}\right)
$$

Thus, $\bar{\Pi}=\left(\begin{array}{cc}0.9833 & 0 \\ 0.0333 & 0.25\end{array}\right)$ and $\rho(\bar{\Pi})<1$.

Theorem 3.2 shows that $\Omega=\left\{\left(x_{1}, x_{2}\right)^{T} \in \mathbb{R}^{2}\left(\begin{array}{c}x_{1} \\ x_{2}\end{array}\right) \leq\left(\begin{array}{c}51.9615 \\ 3.3566\end{array}\right)\right\}$ is a global attracting set of such

system.

\section{Conclusion}

Based on a integral inequality and the property of nonnegative matrix, we obtain some sufficient conditions to ensure the Lagrange stability and the existence of the global attracting set of a class of Hopfield neural networks with delays. The methods of this paper can also be used to study the globally asymptotical stability of equilibrium point. Finally, an example is given to show the effectiveness of our result. 


\section{Acknowledgments}

This work was financially supported by the National Natural Science Foundation of China (No: 11501499, 11271295 and 61573011), the Natural Science Foundation of Guangdong Province (2015A030313707).

\section{References}

[1] M.J. Park, O.M. Kwon, Ju H. Park and S.M. Lee:Expert Syst. Appl. Vol.39 (2012) , p. 5625.

[2] H. Wang, Y.G. Yu and G.G. Wen: Neural Netw. Vol.55 (2014) , p. 98.

[3] H. Wang, Y.G. Yu, G.G. Wen, S. Zhang and J.Z. Yu: Neurocomputing Vol.154(2015) , p. 15.

[4] S. Dharani, R. Rakkiyappan and J.D. Cao: Neurocomputing Vol.151(2015) , p. 827.

[5] B. Song, Y. Zhang, Z. Shu and F.N. Hu: Neurocomputing Vol.196(2016) , p. 53.

[6] S. Zhang, Y.G. Yu and Q. Wang:Neurocomputing Vol.171(2016) , p. 1075

[7] X.X.Liao, Q.Luo and Z.G.Zeng:Neurovomputing Vol.71(2008) , p. 513.

[8] P. Kloeden and D. J. Stonier: Dynam. Contin. Discret. Impul.Syst. Vol.4(1998) , p. 211.

[9] T. Caraballo, P. Marn-Rubio and J. Valero:J. Differ. Equ. Vol.208 (2005) , p. 9.

[10]D.N. Cheban: Bull. Acad. Sci. Rep. Moldova Mat. Vol.2 (1991) , p. 3.

[11] L.X.Zhang, E.K.Boukas and A. Haidar:Automatica Vol.44(2008) , p.2691.

[12] D.Y.Xu:Tohoku Math.J. Vol.44(1992) , p.365.

[13] D.Y.Xu and Z.C.Yang:J.Math.Anal.Appl. Vol.329(2007) , p.1036.

[14] J.P.Lasalle: The stability of dynamical system (Society For Industral and Applied Mathematics Press, Philadelphia, 1976). 